\title{
The Role of SIRT3 in Mediating Cardioprotective Effects of RAS Inhibition on Cardiac Ischemia-Reperfusion
}

\author{
Sabzali Javadov and Nelson Escobales \\ Department of Physiology, School of Medicine, University of Puerto Rico, San Juan, PR, USA \\ Received, June 13, 2015; Revised, October 13, 2015; Accepted, October 13, 2015; Published, October 13, 2015.
}

\begin{abstract}
Cardiac ischemia-reperfusion stimulates the renin-angiotensin system (RAS) associated with elevated levels of circulating angiotensin II. Numerous studies demonstrate that the antagonist for the angiotensin II type 1 receptor, losartan improves cardiac function in animal models of ischemia-reperfusion. Molecular mechanisms of the cardioprotective effects of RAS inhibitors on cardiac ischemia-reperfusion remain poorly understood, and are not associated with the anti-hypertensive action of these drugs. This Commentary focuses on the study published in the Journal of Pharmacy and Pharmaceutical Sciences, 2015, 18:112-123, that elucidates the role of SIRT3 in the cardioprotective action of losartan against ischemic-reperfusion injury. We provide comprehensive discussion of the role of mitochondria in the cardioprotective effects of losartan through SIRT3.
\end{abstract}

This article is open to POST-PUBLICATION REVIEW. Registered readers (see "For Readers") may comment by clicking on ABSTRACT on the issue's contents page.

Cardiac ischemia-reperfusion (IR) is known to stimulate the renin-angiotensin system (RAS) that may have deleterious effects on heart metabolism and function (Figure 1). Activation of RAS during myocardial infarction and ischemic heart disease is associated with elevated levels of circulating angiotensin II (AngII) (1). Also, activation of cardiac RAS in the ischemic myocardium increases intracellular synthesis of AngII that together with circulating AngII exert detrimental effects on cardiac function through autocrine and paracrine mechanisms (2). Notably, short-term treatment with AngII exerts cardioprotective effects on IR similar to those induced by ischemic preconditioning in isolated Langendorff-perfused hearts (3). The deleterious effects of AngII on the ischemic myocardium are mediated through AngII type 1 (AT1) receptors and include suppression of contractility, arrhythmias, alterations of $\mathrm{Ca}^{2+}$ homeostasis and energy metabolism, increased reactive oxygen species (ROS) generation, etc. (4). Consequently, inhibition of AngII production or action in tissues, constitute important therapeutic strategies to protect the heart against IR. Indeed, both angiotensin-converting enzyme (ACE) inhibitors (5), and AT1 receptor blockers have been shown to exert cardioprotection against IR injury (6). Several studies demonstrate that the AT1 receptor antagonist, losartan improves cardiac function in the isolated Langendorff-perfused heart subjected to global IR (7), as well as in in vivo models of IR induced by coronary artery ligation (8). Notably, the cardioprotective effects of RAS inhibitors on cardiac IR are not associated with the anti-hypertensive action of these drugs (9).

Despite the high number of studies available so far, the molecular mechanisms of cardioprotection by RAS inhibition remain unknown. Although blockade of AT1 receptors improves post-ischemic recovery, prevents arrhythmia, increases $\mathrm{Ca}^{2+}$ storage in the sarcoplasmic reticulum, reduces ROS, and attenuates mitochondrial dysfunction, a cause-effect relationship between these effects has not been established. The article by Klishadi and co-authors published in the Journal of Pharmacy and Pharmaceutical Sciences (10) attempts to establish a role for SIRT3 in the cardioprotective action of losartan following IR injury. The authors demonstrated that pre-treatment of rats with losartan $(10 \mathrm{mg} / \mathrm{kg} /$ day) for 4 weeks significantly improved the recovery of hearts after in vivo IR induced by coronary artery ligation $(30 \mathrm{~min})$ and subsequent reperfusion (120 $\mathrm{min})$. They found that electrical heart abnormalities (ventricular tachycardia and ectopic beats) after IR were

Corresponding Author: Sabzali Javadov, Department of Physiology, School of Medicine, University of Puerto Rico, San Juan, PR, USA. E-mail: sabzali.javadov@upr.edu 
attenuated by losartan, a finding that was associated with increased SIRT3 protein levels. The authors concluded that chronic administration of losartan at non-hypotensive levels, could exert cardioprotection in part, through normalization the SIRT3 protein level in the ischemic myocardium (10). However, the involvement and role of mitochondrial SIRT3 in these cardioprotective effects of losartan were not considered, limiting the interpretation of the data.

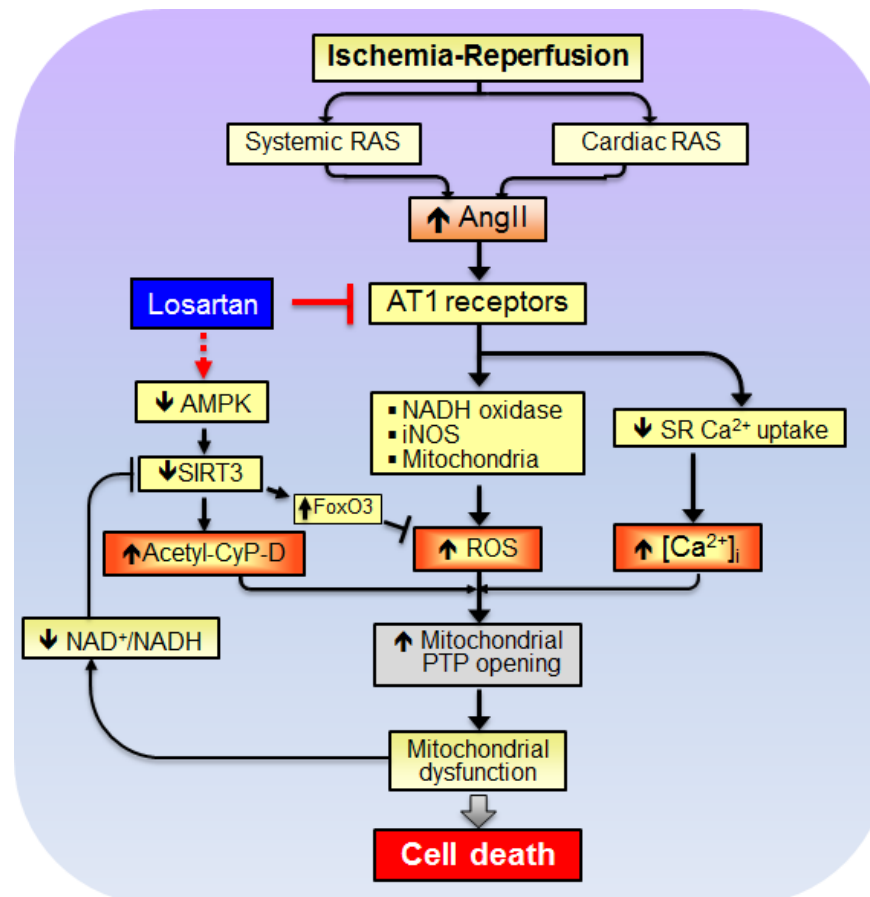

Figure 1. Proposed mechanism of the cardioprotective action of AT1 receptor blockers during ischemiareperfusion. See the text for details. AMPK, AMP kinase; AngII, angiotensin II; AT1, AngII type I; CyP-D, cyclophilin D; FoxO3, forkhead box O3; iNOS, inducible nitric oxide synthase; RAS, renin-angiotensin system, PTP, permeability transition pore; ROS, reactive oxygen species; SR, sarcoplasmic reticulum.

Sirtuins are class III histone deacetylases that depend on $\mathrm{NAD}^{+}$for their activity, and play an essential role in the regulation of protein activity by deacetylation. There are seven sirtuin isoforms (SIRT1-7) which subcellular localization varies between the cytoplasm (SIRT2), nucleus (SIRT1, 6, 7) and mitochondria (SIRT3, 4, 5) (11). Proteomic analysis has identified 277 lysine acetylation sites on 133 mitochondrial proteins, thereby establishing that lysine acetylation is an abundant posttranslational modification in mitochondria (12). Most lysineacetylated proteins $(\sim 100$ proteins $)$ from mitochondrial fractions were metabolic enzymes involved in various aspects of energy metabolism, including the TCA cycle, fatty acid oxidation, and oxidative phosphorylation (13). SIRT3 is the main mitochondrial sirtuin isoform that plays a central role in fatty acid oxidation and ATP synthesis in cells (14). Its expression decreases with age, and neurodegenerative, cardiovascular and metabolic diseases. The study by Klishadi et al (10) did not evaluate mitochondrial function and/or acetylation of mitochondrial proteins in losartan-pretreated versus untreated rats subjected to IR. Also, lack of data on the enzymatic activity of SIRT3 in mitochondria obscures the contribution of SIRT3 to losartan-induced cardioprotection in the ischemic myocardium.

We have previously shown (14) that pretreatment of rats with the direct renin inhibitor, aliskiren $(50 \mathrm{mg} / \mathrm{kg} / \mathrm{day})$ improved cardiac function after permanent coronary artery ligation for four weeks. The beneficial effects of aliskiren were associated with the improved respiratory function of mitochondria and inhibition of mitochondrial permeability pore (PTP) opening. Interestingly, hearts of aliskiren-treated rats demonstrated high SIRT3 levels and decreased acetylation of mitochondrial proteins including cyclophilin D (CyP-D), a key regulator of PTP formation (15). These data suggest that chronic inhibition of RAS could exert cardioprotective actions through inhibition of PTP formation by SIRT3-mediated deacelylation of CyP-D.

Chronic blockade of AT1 receptors with losartan could also reduce damaging autocrine/paracrine effects of AngII on coronary arteries and myocardium. Losartan-induced vasodilatation could improve oxygen and substrate delivery to the ischemic myocardium at reperfusion. In addition, inhibition of AT1 receptor by losartan could prevent ROS accumulation by NADH-oxidase (4), inducible nitric oxide synthase (iNOS) (16) and mitochondria $(17,18)$ in cardiac cells. A role of losartan in maintaining intracellular $\mathrm{Ca}^{2+}$ homeostasis in isolated guinea pig ventricular myocytes following IR injury has been proposed (19). Since ROS and $\mathrm{Ca}^{2+}$ are the main inducers of mitochondrial PTP, reductions in their levels by losartan following IR could prevent pore opening and improve mitochondrial function and ATP production. The latter could lead to a reduction in the AMP to ATP ratio and stimulation of AMP kinase (AMPK), a serine/threonine kinase that acts as a "fuel sensor" 
and regulates energy metabolism in the heart. Activation of AMPK is known to stimulate ATP synthesis, glucose transport, glycolysis and fatty acid oxidation, and inhibits energy-consuming anabolic pathways such as protein synthesis (20). Indeed, we have shown that losartan enhanced AMPK phosphorylation in AngII-treated cardiomyocytes (17). Losartan-induced activation of AMPK could upregulate SIRT3 activity through changes in the $\mathrm{NAD}^{+} / \mathrm{NADH}$ ratio that is the main regulator of sirtuins. AMPK-dependent increases in protein expression of SIRT3 and manganese superoxide dismutase (MnSOD) were found in the mouse skeletal muscle (21). Interestingly, the beneficial effects of SIRT3 can be mediated through a direct upregulation of antioxidant capacity of cardiomyocytes. SIRT3 has been shown to induce deacetylation and translocation of the forkhead box O3 (FoxO3), a transcription factor, to the nucleus, where it activates antioxidant-encoding genes such as MnSOD and catalase, thereby decreasing cellular levels of ROS (22). Also, SIRT3 can stimulate PGC$1 \alpha$ and its downstream targets that regulate mitochondrial biogenesis and play a crucial role in cardiac diseases (23).

It is likely that acetylation of CyP-D due to downregulation of SIRT3 facilitates its interaction with the PTP complex and stimulate pore opening leading to mitochondria-mediated cell death and cardiac dysfunction. A causal role of CyP-D acetylation induced by downregulation of SIRT3 in mitochondrial PTP opening was demonstrated previously (24). As mentioned above, aliskiren prevented CyP-D acetylation that was associated with upregulation of SIRT3 expression and PTP inhibition in post-infarction rat hearts (15). Notably, the beneficial effects of losartan on mitochondria can also be mediated through AngII receptors present in mitochondria. We (15) and others (25) reported the expression of AT1 and Ang II type 2(AT2) receptors in cardiac and kidney mitochondria. In addition, a role for AT2 receptor activation in losartan-mediated cardioprotection cannot be excluded in the setting of RAS activation. This point needs to be evaluated. In addition to acetylation of CyP-D due to downregulation of SIRT3, cardiac IR can activate CyP-D through its interaction with the peroxisome proliferator-activated receptor alpha (PPAR $\alpha)$. We have recently shown that the PPAR $\alpha / \mathrm{CyP}-\mathrm{D}$ was associated with PTP opening in cultured cardiomyocytes subjected to oxidative stress [26] and in vivo cardiac IR [27]. Activation of AMPK by metformin abrogated the interaction and prevented PTP opening in both cases.

In conclusion, the study presented by Klishadi and coauthors (10) is an interesting study that attempts to elucidate the role of AngII/AT1 receptors/SIRT3 pathway in losartan-induced cardioprotection against IR injury. This report together with previous studies indicates the importance of mitochondria in attenuation of cardiac dysfunction by the chronic use of RAS inhibitors in response to oxidative stress.

\section{ACKNOWLEDGMENTS}

This work was supported by the National Heart, Lung, And Blood Institute of the National Institutes of Health through Research Grant SC1HL118669 (S.J.)

\section{REFERENCES}

1. Ertl G, Hu K. Anti-ischemic potential of drugs related to the renin-angiotensin system. J Cardiovasc Pharm, 2001; 37 Suppl 1:S11-20.

2. So T, Nakashima $Y$, Imamura M, Arakawa K. Effects of local inhibition of the cardiac renin-angiotensin system with CV-11974 in a canine ischaemiareperfusion model. Clin Exp Pharmacol Physiol, 1998; 25:503-509.

3. Nunez RE, Castro M, Javadov S, Escobales N. Angiotensin II and ischemic preconditioning synergize to improve mitochondrial function while showing additive effects on ventricular postischemic recovery. J Cardiovasc Pharm, 2014; 64:172-179.

4. Schmidt-Ott KM, Kagiyama S, Phillips MI. The multiple actions of angiotensin II in atherosclerosis. Regul Peptides, 2000; 93:65-77.

5. Pfeffer MA. Enhancing cardiac protection after myocardial infarction: rationale for newer clinical trials of angiotensin receptor blockers. Am Heart J, 2000; 139:S23-28.

6. Schulz R, Heusch G. AT1-receptor blockade in experimental myocardial ischemia/reperfusion. Clin Nephrol, 2003; 60 Suppl 1:S67-74.

7. Paz Y, Gurevitch J, Frolkis I, Matsa M, Kramer A, Locker C, Mohr R, Keren G. Effects of an angiotensin II antagonist on ischemic and nonischemic isolated rat hearts. Ann Thorac Surg, 1998; 65:474-479.

8. Yahiro E, Ideishi M, Wang LX, Urata H, Kumagai K, Arakawa K, Saku K. Reperfusion-induced arrhythmias are suppressed by inhibition of the angiotensin II type 1 receptor. Cardiology, 2003; 99:61-67. 
9. Dorn GW, 2nd. Novel pharmacotherapies to abrogate postinfarction ventricular remodeling. Nat Rev Cardiol, 2009; 6:283-291.

10. Klishadi MS, Zarei F, Hejazian SH, Moradi A, Hemati M, Safari F. Losartan protects the heart against ischemia reperfusion injury: sirtuin3 involvement. J Pharm Pharm Sci, 2015; 18:112-123.

11. Guarente L. Sirtuins as potential targets for metabolic syndrome. Nature, 2006; 444:868-874.

12. Kim SC, Sprung R, Chen Y, Xu Y, Ball H, Pei J, Cheng T, Kho Y, Xiao H, Xiao L, Grishin NV, White $\mathrm{M}$, Yang XJ, Zhao Y. Substrate and functional diversity of lysine acetylation revealed by a proteomics survey. Mol Cell, 2006; 23:607-618.

13. Mootha VK, Bunkenborg J, Olsen JV, Hjerrild M, Wisniewski JR, Stahl E, Bolouri MS, Ray HN, Sihag S, Kamal M, Patterson N, Lander ES, Mann M. Integrated analysis of protein composition, tissue diversity, and gene regulation in mouse mitochondria. Cell, 2003; 115:629-640.

14. Ahn BH, Kim HS, Song S, Lee IH, Liu J, Vassilopoulos A, Deng CX, Finkel T. A role for the mitochondrial deacetylase Sirt3 in regulating energy homeostasis. Proc Natl Acad Sci USA, 2008; 105:14447-14452.

15. Parodi-Rullan R, Barreto-Torres G, Ruiz L, Casasnovas J, Javadov S. Direct renin inhibition exerts an anti-hypertrophic effect associated with improved mitochondrial function in post-infarction heart failure in diabetic rats. Cell Physiol Biochem, 2012; 29:841-850.

16. de Gusmao FM, Becker C, Carvalho MH, Barros LF. Angiotensin II inhibition during myocardial ischemia-reperfusion in dogs: effects on leukocyte infiltration, nitric oxide synthase isoenzymes activity and left ventricular ejection fraction. Int $\mathrm{J}$ Cardiol, 2005; 100:363-370.

17. Hernandez JS, Barreto-Torres G, Kuznetsov AV, Khuchua Z, Javadov S. Crosstalk between AMPK activation and angiotensin II-induced hypertrophy in cardiomyocytes: the role of mitochondria. J Cell Mol Med, 2014; 18:709-720.

18. McLachlan J, Beattie E, Murphy MP, Koh-Tan CH, Olson E, Beattie W, Dominiczak AF, Nicklin SA, Graham D. Combined therapeutic benefit of mitochondria-targeted antioxidant, MitoQ10, and angiotensin receptor blocker, losartan, on cardiovascular function. J Hypertens, 2014; 32:555564.

19. Louch WE, Ferrier GR, Howlett SE. Attentuation of cardiac stunning by losartan in a cellular model of ischemia and reperfusion is accompanied by increased sarcoplasmic reticulum $\mathrm{Ca} 2+$ stores and prevention of cytosolic $\mathrm{Ca} 2+$ elevation. J Pharmacol Exp Ther, 2005; 312:238-247.
20. Arad M, Seidman CE, Seidman JG. AMP-activated protein kinase in the heart: role during health and disease. Circ Res, 2007; 100:474-488.

21. Brandauer J, Andersen MA, Kellezi H, Risis S, Frosig C, Vienberg SG, Treebak JT. AMP-activated protein kinase controls exercise training- and AICARinduced increases in SIRT3 and MnSOD. Front Physiol, 2015; 6:85.

22. Sundaresan NR, Gupta M, Kim G, Rajamohan SB, Isbatan A, Gupta MP. Sirt3 blocks the cardiac hypertrophic response by augmenting Foxo3adependent antioxidant defense mechanisms in mice. $\mathrm{J}$ Clin Invest, 2009; 119:2758-2771.

23. Javadov S, Purdham DM, Zeidan A, Karmazyn M. NHE-1 inhibition improves cardiac mitochondrial function through regulation of mitochondrial biogenesis during postinfarction remodeling. Am J Physiol Heart Circ Physiol, 2006; 291:H1722-1730.

24. Shulga N, Pastorino JG. Ethanol sensitizes mitochondria to the permeability transition by inhibiting deacetylation of cyclophilin-D mediated by sirtuin-3. J Cell Sci, 2010; 123:4117-4127.

25. Abadir PM, Foster DB, Crow M, Cooke CA, Rucker JJ, Jain A, Smith BJ, Burks TN, Cohn RD, Fedarko NS, Carey RM, O'Rourke B, Walston JD. Identification and characterization of a functional mitochondrial angiotensin system. Proc Natl Acad Sci USA, 2011; 108:14849-14854.

26. Barreto-Torres G, Hernandez JS, Jang S, RodríguezMuñoz AR, Torres-Ramos CA, Basnakian AG, Javadov S. The beneficial effects of AMP kinase activation against oxidative stress are associated with prevention of PPAR $\alpha$-cyclophilin D interaction in cardiomyocytes. Am J Physiol Heart Circ Physiol. 2015; 308:H749-H758.

27. Barreto-Torres G, Parodi-Rullán R, Javadov S. The role of PPAR $\alpha$ in metformin-induced attenuation of mitochondrial dysfunction in acute cardiac ischemia/reperfusion in rats. Int $\mathrm{J}$ Mol Sci. 2012;13:7694-709. 